\title{
An Integral Field Unit Based on Parabolic Mirror ${ }^{\star}$
}

\author{
Mariia Orekhova ${ }^{[0000-0002-4814-2847]}$ and \\ Alexey Bakholdin ${ }^{[0000-0002-7788-1593]}$ \\ ITMO University, St. Petersburg, Russia \\ mkorekhova@itmo.ru, bakholdinditmo.ru
}

\begin{abstract}
Recently many modern instruments and systems have been developed to study the Sun. For that, spectral instruments with high spectral resolution are most often used. It is relevant to achieve high spatial resolution along with spectral one for many scientific tasks. In practice, the achievement of both high spectral and spatial resolution can be done by the use of integral field spectroscopy.

Current paper is devoted to searching for a system solution for an integral field unit (IFU), which will be implemented to the optical system of solar telescopecoronagraph. The diameter of the main mirror is $D=3 \mathrm{~m}$. Telescope's working spectral range is $\Delta \lambda=390-1600 \mathrm{~nm}$.

The integral field unit is based on reflective elements. It divides the input field of a rectangular shape with a size of $0.75^{\prime \prime} \times 12^{\prime \prime}(0.145 \mathrm{~mm} \times 2.327 \mathrm{~mm})$ into 8 parts with a size of $0.094^{\prime \prime} \times 96^{\prime \prime}(0.018 \mathrm{~mm} \times 18.617 \mathrm{~mm})$ each. The possibility of creating an IFU optical system using a parabolic mirror for all (eight) channels is shown. The quality of the optical system was evaluated, as well as the effect of vignetting on the slicing mirrors.
\end{abstract}

Keywords: Integral Field Spectroscopy, High Spatial Resolution, Solar Telescope.

\section{Introduction}

Currently, the development and refinement of the characteristics of the KST-3 solar telescope-coronograph [1-2] with the diameter of the main mirror $D=3 \mathrm{~m}$ is being conducted. The equipment will allow observation and measurement of the solar structure with high spectral $(R \sim 300,000)$ and spatial $\left(0.1^{\prime \prime}\right)$ resolution.

The solar telescope-coronagraph's characteristics are presented in Table 1.

It is relevant to achieve high spatial resolution, along with spectral one, in astronomical optical devices. However, classic spectroscopy can only provide high spectral resolution. The task of achieving high spatial resolution can be solved by building a spectral block by using the integral field spectroscopy technique.

Integral field spectroscopy is an area in astronomy that allows to collect information about the spectrum of a two-dimensional field with high spectral resolution and the spatial one.

Copyright $\odot 2020$ for this paper by its authors. Use permitted under Creative Commons License Attribution 4.0 International (CC BY 4.0).

* Publication is supported by RFBR grant 19-01-00435 
Table 1. The main characteristics of the telescope

\begin{tabular}{|c|c|}
\hline The field of a rectangular shape & $6^{\prime \prime} \times 12^{\prime \prime}$ \\
\hline Focal lengh & $40 \mathrm{~m}$ \\
\hline Aperture & 0,075 \\
\hline Spectral range & $0,39-1,60 \mu \mathrm{m}$ \\
\hline
\end{tabular}

Schematically, the method of integral field spectroscopy is presented in Figure 1. The Gregorian mirror system 2 [1-2] forms an image of the Sun's part 1 with a diameter of $2^{\prime}$. The field stop 3 in front of the integral field unit 4 (IFU) "cuts" a $1.2 \mathrm{~mm} \times 2.3 \mathrm{~mm}$ rectangular field in the focal plane of the telescope, which corresponds to an angular size of $6^{\prime \prime} \times 12^{\prime \prime}$. Further, the integral field unit 4 transforms a two-dimensional rectangular field into a set of long slits 5 , which are the object for a spectrometer 6 . The spectrometer 6 forms spectral images 7 of a slit 5 in the spectral range of $0.39-1.60 \mu \mathrm{m}$.

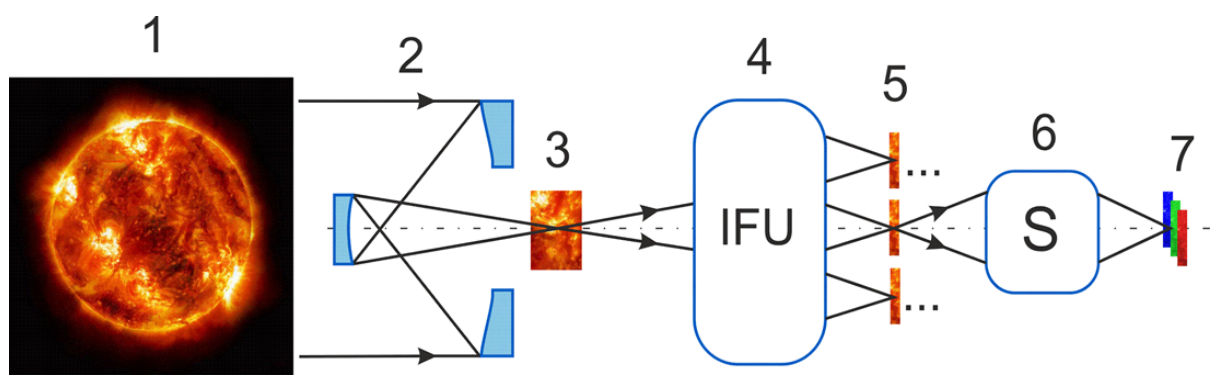

Fig. 1. Integral field spectroscopy: 1 - the Sun, 2 - the Gregorian telescope, 3 - field stop, 4 integral field unit, 5 - a set of longslits. 6 - a spectrometer, 7 - longslits' spectral images

Today, projects such as MuSICa [3-5], MUSE [6-10], FISICa [11-12], developed by the use of integral field spectroscopy, merely begin to be implemented. There are no such implemented systems for studying the Sun in a wide spectral range $(0.39-$ $1.60 \mu \mathrm{m})$.

\section{Image Slicer}

A distinctive feature of the spectral block, constructed using integral field spectroscopy, is the implementation of the integral field unit 4 into the optical system (Figure 1). The main element of such a system is an image slicer, which is installed in the focal plane of the telescope and divides the field into the desired number of slices. It can be designed based on [13-15]:

- an array of microlenses,

- an optical fiber,

- an array of small-width mirrors. 
In this work, since the device should operate in a wide spectral range, it is advisable to use an array of reflective elements to cut the field. Moreover, it is a compact, elegant and highly efficient solution that can eliminate many of the critical drawbacks of other alternative designs for an IFU. When using a mirror image slicer, there is no overlapping of the spectra, as in the case of microlenses, and it does not have the disadvantages associated with optical fibers, such as a deterioration of the focal ratio, transmission loss of infrared wavelengths and the depolarization nature of the fibers as a whole.

An IFU principal scheme with an array of plane mirrors as an image slicer is shown in Figure 2. The diverging light beam 1 from the focal plane of the telescope is divided into parts by an array of plane mirrors 2 (image slicer). Those mirrors are tilted in different directions. Further, focusing mirrors 3 forms an image of the slit in the plane 4. The image 4 is fed to the input of a spectrometer, forming spectral images of the slit.

The axis on which the array of mirrors of the image slicer 2 is located is perpendicular to the plane of the figure.

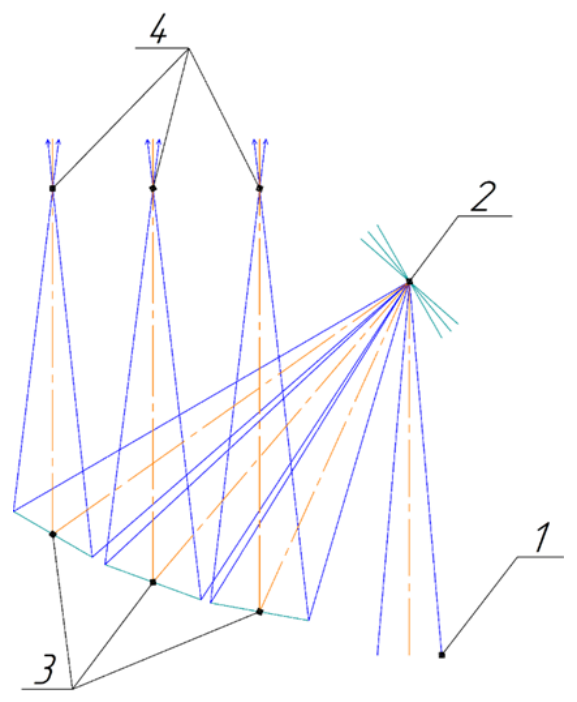

Fig. 2. Image slicer: 1 - light beam, 2 - image slicer, 3 - focusing mirrors. 4 - longslit

\section{The System Solution of an Integral Field Unit}

In the current paper, the angular size of the rectangular input field is $6^{\prime \prime} \times 12^{\prime \prime}$. It is necessary to provide a resolution limit of $0.1^{\prime \prime}$ at the output of the optical system. Thus, dividing the input field by 64 slices is sufficient, which allows to get a final slit of size $0.094^{\prime \prime}$. At the first stage the rectangular field is divided into 8 parts, and at the second stage - into another 8 . 
Modeling of the second stage of the integral field module is shown, since the rectangular field is reorganized into a narrow slit on the second stage.

Figure 3 shows a system solution of the second stage IFU. The light beam from the telescope 1 is focused in its focal plane 2, where an image slicer is installed (the figure shows only one channel). Further, the reflected light beam enters the parabolic mirror 3. The parallel beam is directed to a system of flat mirrors $4-5$, which work like a scanning system and serve to reorganize a rectangular image into a narrow slit. After reflection from the mirror 5 , the light beam is focused by a paraxial lens 6 in its focal plane 7 .

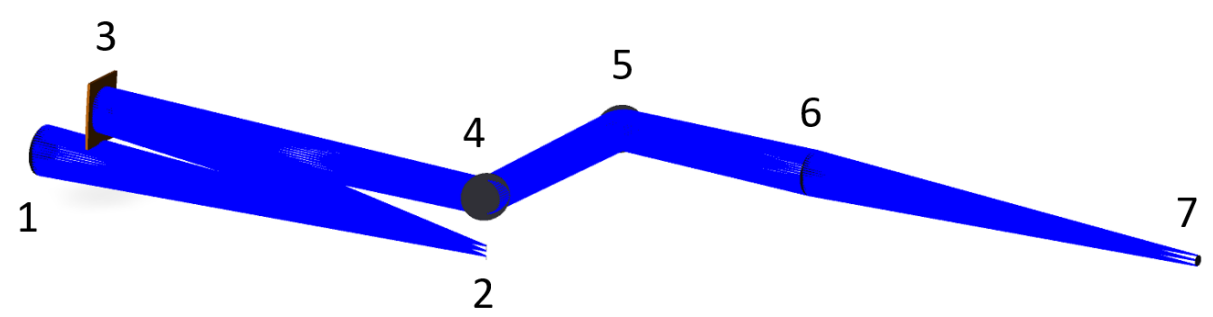

Fig. 3. The system solution of an integral field unit (the only one channel is presented)

Figure 4 shows the IFU system solution with all channels. A single parabolic mirror is used for collimation, as if all the channels made up a single light beam. This approach makes it possible to collect images of all channels in one plane, and provides a minimum gap between images. In addition, another advantage of using a parabolic surface is the equal directivity of the beam apertures of all channels, which is important for connecting with the next block (spectral).

For transforming a rectangular image into a narrow slit, it is necessary that the images of all slices have gaps between them in the meridional plane. In the sagittal plane they should form a straight line. The first requirement is achieved by arranging the elements as shown in Figures 3-4. To fulfill the second requirement, it is necessary to achieve the displacement of the slice images due to the rotation of the flat mirror 5 (Figure 3) along the Y axis. The rotation of the flat mirror is calculated by the following equation:

$$
\alpha=\frac{a}{b}
$$

where $a$ is a linear coordinate of the slice central point, $b$ is the distance from paraxial lens 6 (Figure 3) to the image plane.

Angles of each mirrors' tilt in respect to the telescope optical axis are shown in Table 2. These values represent the minimum values for the effective positioning of all the elements in space. The width of each mirror is equal to $18 \mu \mathrm{m}$. Figure 5 shows the image slicer with four mirrors, since the other four are tilted by the same degree, but in the opposite direction.

Figure 6 shows the spot diagrams for eight channels and three points of the field of the integral field unit. The discrepancy between the spots of the extreme points of the field of different channels is explained by the use of a common parabolic mirror: for 


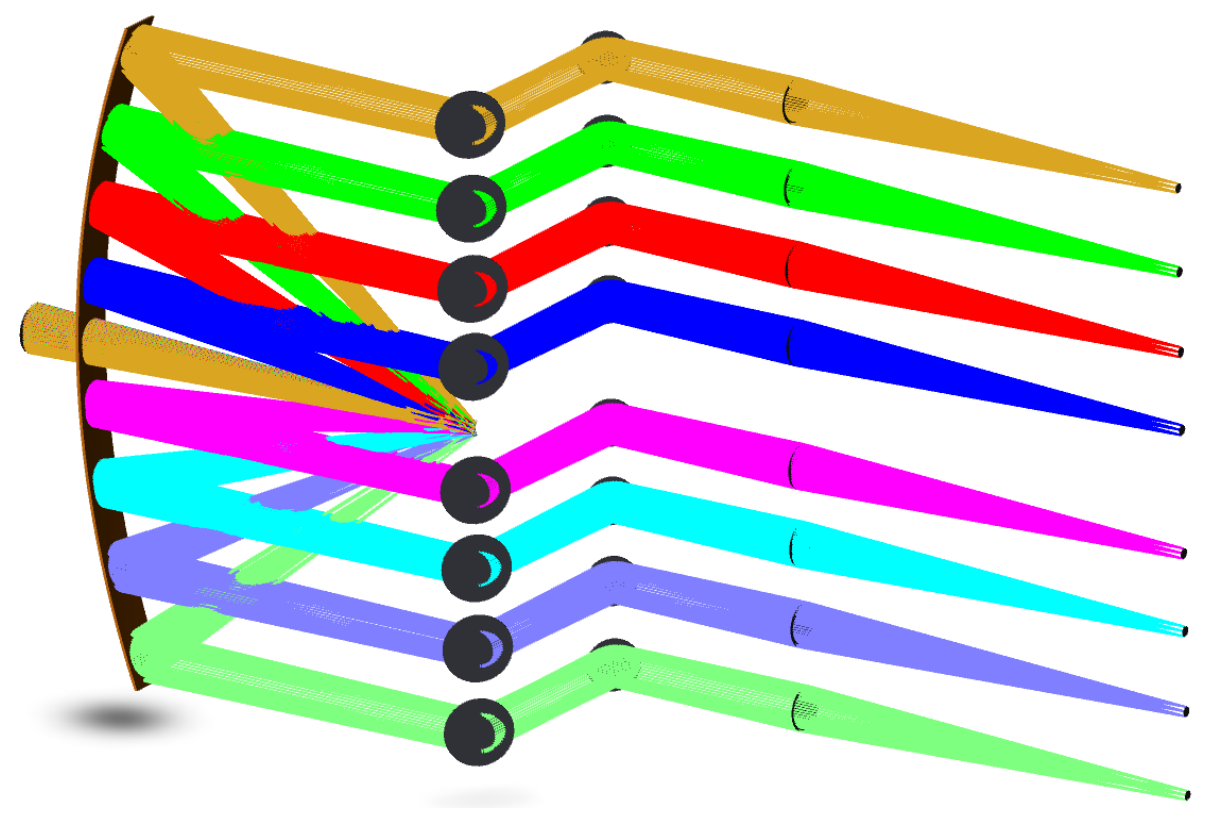

Fig. 4. The system solution of an integral field unit (all the channels)

Table 2. The main characteristics of the telescope

\begin{tabular}{|c|c|c|c|c|c|c|c|c|}
\hline Mirror's number & 1 & 2 & 3 & 4 & 5 & 6 & 7 & 8 \\
\hline Tilt, angular degrees & 14 & 10,5 & 7 & 3,5 & $-3,5$ & -7 & $-10,5$ & -14 \\
\hline
\end{tabular}

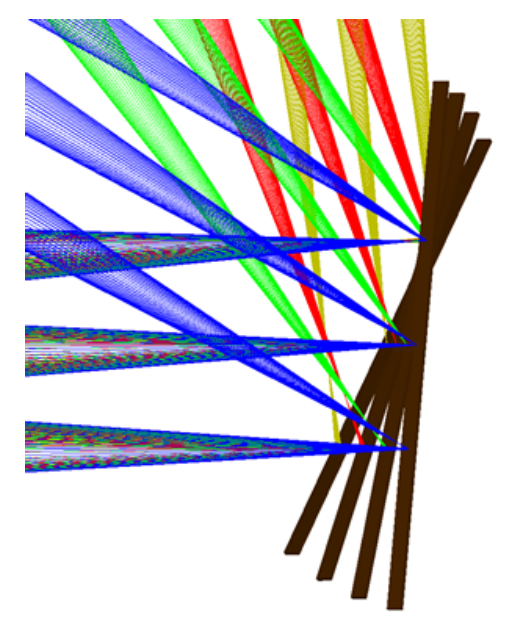

Fig. 5. Image slicer (four mirrors are presented) 
different zones of this element, the distances from the focal plane are not equidistant. This is the disadvantage of using a single surface for collimating beams of all channels.

Also, it can be seen that the light beams of the extreme points of the field are vignetted. This situation is explained by the fact that the beam aperture exceeds the angular size of the image slicer.

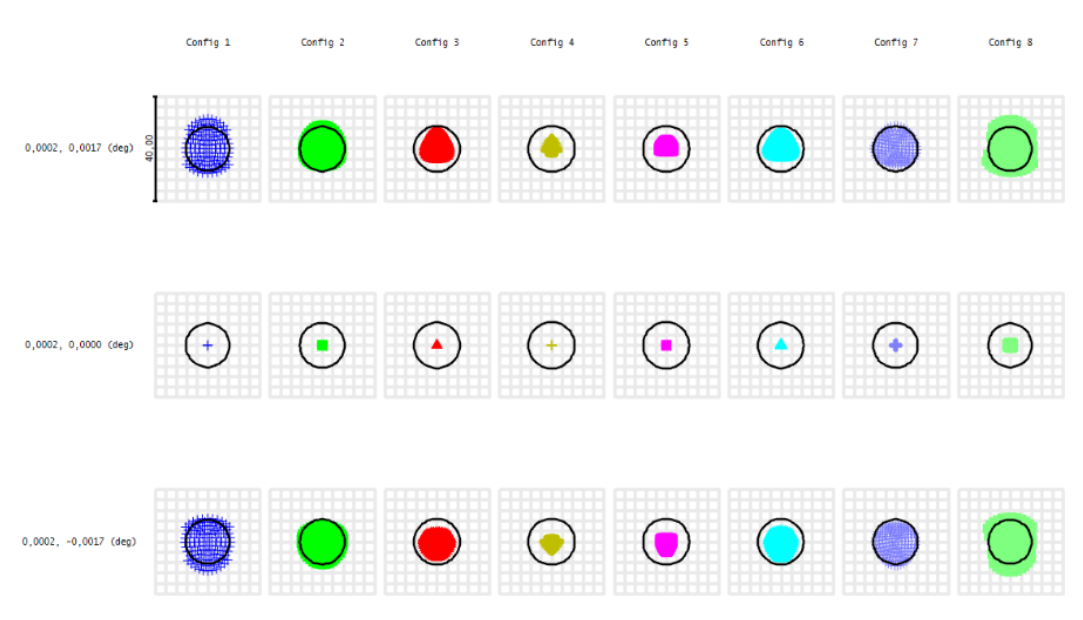

Fig. 6. Spot diagrams

\section{Conclusion}

The paper shows the possibility of designing an optical system for an integral field unit based on a single reflective element for several channels. The concept of integral field spectroscopy is explained, a schematic diagram of the technique is proposed. The classification of optical elements performing image slicing is shown, and the choice of an array of small-width mirrors for this problem is justified.

A system solution of the integral field unit is shown for dividing the input field of a rectangular shape $0.75^{\prime \prime} \times 12^{\prime \prime}$ in size $(0.145 \mathrm{~mm} \times 2.327 \mathrm{~mm})$ into 8 parts of $0.094^{\prime \prime} \times 96^{\prime \prime}$ in size $(0.018 \mathrm{~mm} \times 18.617 \mathrm{~mm})$ each. The quality of the resulting system solution is estimated by diffraction limit on aperture stop and by vignetting and diffraction effects on image slicer. Using the presented system solution, it is possible to develop an integral field unit for the use in astronomical optics systems, as well as for other applications.

\section{References}

1. Demidov M. et al.: Proekt natsionalnogo rossiiskogo krupnogo solnechnogo telescopa s diametrom zerkala $3 \mathrm{~m}$. In: X ROSSIISKO-MONGOLSKAYA CONFERENTSIA "SOLNECHNO-ZEMNAYA PHISICA I SEISMOGEODINAMIKA BAIKALOMONGOLSKOGO REGIONA”, pp. 55-67. Mongolia (2014). 
2. Kolobov D. et al.: Natsionalnyi proekt krupnogo solnechnogo telescopa: zadachi i perspektivy. In: MEJDUNARODNAYA BAIKALSKAYA MOLODEJNAYA NAUCHNAYA SHKOLA PO FUNDAMENTALNOI PHISIKE I XIV KONFERENCIA MOLODYX UCHENYH "VZAIMODEISTVIE POLEI I IZLUCHENIA S VESHESTVOM", pp.3-4. Irkutsk (2015).

3. Calcines, A. et al.: MuSICa: the Multi-Slit Image Slicer for the EST Spectrograph. Journal of Astronomical Instrumentation 2(1), 135000 (2013).

4. Calcines, A. et al: Feasibility study of high-resolution integral-field spectrographs for EST with multislit and multi-wavelength capabilities. In: Ground-based and Airborne Instrumentation for Astronomy III, pp. 77351X. Proc. SPIE (7735), USA (2010).

5. Calcines, A. et al.: MuSICa image slicer prototype at 1.5-m GREGOR solar telescope. In: Ground-based and Airborne Instrumentation for Astronomy V, pp. 91473I. Proc. SPIE (9147), USA (2014).

6. Henault F. et al.: Slicing the universe at affordable cost: the quest for the MUSE image slicer. In: Optical Design and Engineering, pp. 134-145. Proc. SPIE (5249), France (2004).

7. Laurent F. et al.: Optical design, manufacturing, and tests of the MUSE image slicer. In: Optical Fabrication, Testing, and Metrology II, pp. 59650J. Proc. SPIE (5965), USA (2005).

8. Laurent F. et al.: MUSE Integral Field Unit: Test results on the first out of 24. In: SPIE Astronomical Telescopes + Instrumentation, pp. 77394M. Proc. SPIE (7739), USA (2010).

9. Laurent F. et al.: MUSE field splitter unit: fan-shaped separator for 24 integral field units. In: Advances in Optical and Mechanical Technologies for Telescopes and Instrumentation, pp. 91511U. Proc. SPIE (9151), USA (2014).

10. Bonneville, C. et al.: Image slicer manufacturing: from space application to mass production. In: Optical Fabrication, Metrology, and Material Advancements for Telescopes, pp. 188 - 195. Proc. SPIE (5494), USA (2004).

11. Stephen S. Eikenberry et al.: FISICA: the Florida image slicer for infrared cosmology and astrophysics. In: Ground-based Instrumentation for Astronomy, pp. 1264 - 1273. Proc. SPIE (5492), USA (2004).

12. Paul E. Glenn et al.: Design, fabrication, assembly, and testing of the Florida image slicer for infrared cosmology and astrophysics (FISICA) integral field unit. In: Ground-based Instrumentation for Astronomy, pp. 1254 - 1263. Proc. SPIE (5492), USA (2004).

13. Bacon R., Monnet G.: Optical 3D-Spectroscopy for Astronomy. John Wiley \& Sons, USA (2017).

14. Mediavilla E. et al.: 3D Spectroscopy in Astronomy (17). Cambridge University Press, USA (2010).

15. Allington-Smith J.: Basic principles of integral field spectroscopy. New Astronomy Reviews 50(4-5), 244-251 (2006). 